\title{
A study of the mechanical properties of as-received and intraorally exposed single-crystal and polycrystalline orthodontic ceramic brackets
}

\begin{abstract}
Alexopoulou, Eleni ; Polychronis, Georgios ; Konstantonis, Dimitrios ; Sifakakis, Iosif ; Zinelis, Spiros ; Eliades,
\end{abstract} Theodore

\begin{abstract}
BACKGROUND: Although ceramic brackets have been extensively used for decades in orthodontics there is not till today any study focusing on the possible deterioration of mechanical properties after in vivo ageing. OBJECTIVES: To determine whether the mechanical properties of alumina orthodontic brackets change after intraoral ageing thereby assessing the validity of a theoretical model established for the performance of ceramics in wet environments. MATERIALS AND METHODS: Two alumina brackets, one single crystal (Radiance, American Orthodontics, Sheboygan, WI) and one polycrystalline (Clarity, 3M, St. Paul, MN) were included in this study. Ten brackets for each group were collected from different patients after a minimum of 3-month intraoral exposure, whereas as-received brackets of the same manufacturers were used as controls. The specimens were subjected to Raman spectroscopy and were then embedded in epoxy resin and metallographic ground and polished. The mechanical properties of four groups (radiance control: RAC, radiance-retrieved RAR, clarity control: CLC and clarity-retrieved CLR) were determined using instrumented indentation testing according to ISO 14577-2002. The mechanical properties tested were Martens hardness (HM), indentation modulus (EIT), the ratio of elastic to total work, commonly known as elastic index ( $\eta \mathrm{IT})$, and fracture toughness (KIC). The numerical results were statistically analysed employing two-way analysis of variance (ANOVA) and Tukey multiple comparison test at $\mathrm{a}=0.05$. RESULTS: Raman analysis revealed that both brackets are made of a-Al2O3 (corundum). No statistically significant differences were found for HM (N/mm2): RAC = 7249 (1507), RAR = 6926 (1144), CLC $=8052$ (1360), CLR = 7390 (2393), or for EIT $(\mathrm{GPa}): \mathrm{RAC}=141$ (27), RAR = 139 (23), CLC = 139 (28), CLR = 131 (47). However, significant differences were identified between the two alumina brackets tested for $\eta \mathrm{IT}(\%)$ : RAC $=55.7$ (4.2), RAR $=54.0$ (3.5), CLC = 62.5 (4.4), CLR = 61.8 (4.7), while KIC was measured only for the polycrystalline bracket (Clarity) because of the complicated fractured pattern of the single-crystal bracket. Both brackets share equal HM and EIT before and after orthodontic intraoral ageing. LIMITATIONS: Whereas the study assessed the changes after intraoral exposure per theoretical model, which describes the reduction of critical stress to induce fracture after wetting, long-term intraoral ageing could have induced more pronounced effects. CONCLUSIONS/IMPLICATIONS: The results of this study indicate that 3 months of intraoral ageing do not change the mechanical properties of single-crystal and polycrystalline orthodontic brackets tested, thus indicating that the Griffith theory may not be applied to the case of manufactured ceramic brackets owing possibly to internal defects.
\end{abstract}

DOI: https://doi.org/10.1093/ejo/cjz024

Posted at the Zurich Open Repository and Archive, University of Zurich

ZORA URL: https://doi.org/10.5167/uzh-191211

Journal Article

Accepted Version 
Originally published at:

Alexopoulou, Eleni; Polychronis, Georgios; Konstantonis, Dimitrios; Sifakakis, Iosif; Zinelis, Spiros; Eliades, Theodore (2020). A study of the mechanical properties of as-received and intraorally exposed single-crystal and polycrystalline orthodontic ceramic brackets. European Journal of Orthodontics, 42(1):72-77.

DOI: https://doi.org/10.1093/ejo/cjz024 
A study of the mechanical properties of as-received and intraorally-exposed single-crystal and polycrystalline orthodontic ceramic brackets

Eleni Alexopoulou, ${ }^{1}$ Georgios Polychronis, ${ }^{2}$ Dimitrios Konstantonis, ${ }^{3}$ Iosif Sifakakis, ${ }^{3}$ Spiros Zinelis, ${ }^{2}$ and Theodore Eliades ${ }^{1}$

\section{Eur J Orthod. 2020 Jan 27;42(1):72-77. doi: 10.1093/ejo/cjz024.}

${ }^{1}$ Clinic of Orthodontics and Pediatric Dentistry, Center of Dental Medicine, University of Zurich, Zurich, Switzerland.

${ }^{2}$ Department of Dental Biomaterials, School of Dentistry, National and Kapodistrian University of Athens, Athens, Greece.

${ }^{3}$ Department of Orthodontics, School of Dentistry, National and Kapodistrian University of Athens, Greece.

Corresponding author:

Theodore Eliades, Clinic of Orthodontics and Pediatric Dentistry, Center of Dental Medicine, University of Zurich,

Plattenstrasse 11, Zurich 8032, Switzerland

(e-mail: theodore.eliades@zzm.uzh.ch) 


\section{Summary}

Objectives: To determine if the mechanical properties of alumina orthodontic brackets change after intraoral aging thereby assessing the validity of a theoretical model established for the performance of ceramics in wet environments.

Materials and methods: Two alumina brackets, one single crystal (Radiance, American Orthodontics, Sheboygan, WI) and one polycrystalline (Clarity, 3M, St. Paul, MN) were included in this study. Ten brackets for each group were collected from different patients after a minimum of 3 months intraoral exposure whereas as-received brackets of same manufacturers were used as controls. The specimens were subjected to Raman spectroscopy and were then embedded in epoxy resin and metallographic ground and polished. The mechanical properties of four groups (radiance control: RAC, radiance retrieved RAR, clarity control: $C L C$ and clarity retrieved $C L R$ ) were determined using instrumented indentation testing according to ISO 14577-2002. The mechanical properties tested were Martens hardness $(\mathrm{HM})$, indentation modulus $\left(\mathrm{E}_{\mathrm{IT}}\right)$ the ratio of elastic to total work, commonly known as elastic index $\left(\eta_{I T}\right)$ and fracture toughness $K_{\mathrm{IC}}$. The numerical results were statistically analyzed employing two way ANOVA and Tukey multiple comparison test at a=0.05.

Results: Raman analysis revealed that both brackets are made of a-Al2O3 (corundum). No statistically significant differences were found for $\mathrm{HM}\left(\mathrm{N} / \mathrm{mm}^{2}\right)$ : RAC=7249 (1507), RAR=6926 (1144), $C L C=8052$ (1360), $C L R=7390$ (2393), or for $E_{\mid T}(G P a): R A C=141$ (27), RAR=139 (23), $C L C=139$ (28), $C L R=131$ (47). However significant differences were identified between the two alumina brackets tested for $\eta_{\text {IT }}(\%)$ : $R A C=55.7$ (4.2), $R A R=54.0$ (3.5), $C L C=62.5$ (4.4), $C L R=61.8$ (4.7), whilst $K_{\mathrm{IC}}$ was measured only for the polycrystalline bracket (Clarity) because of the complicated fractured pattern of the single-crystal bracket. Both brackets share equal $\mathrm{HM}$ and $\mathrm{E}_{\mathrm{IT}}$ before and after orthodontic intraoral ageing. 
Limitations: Whereas the study assessed the changes after intraoral exposure per theoretical model which describes the reduction of critical stress to induce fracture after wetting, long-term intraoral ageing could have induced more pronounced effects.

Conclusions: The results of this study indicate that four months of intraoral ageing does not change the mechanical properties of single-crystal and polycrystalline orthodontic brackets tested thus indicating that the Griffith theory may not be applied to the case of manufactured ceramic brackets owing possibly to internal defects. 


\section{Introduction}

After almost four decades from their appearance in orthodontic clinical practice, single-crystal and polycrystalline alumina and zirconia ceramic brackets, possess a central position in the armamentarium of aesthetic orthodontic materials (1-4). Although ceramic brackets are free of stain and discoloration complications of their plastic counterparts, the increased fracture incidence and complicated debonding process of alumina brackets precluded their wide scale application in everyday practice $(5,6)$. The use of zirconia brackets is limited due to increased friction coefficient, worse aesthetics and decreased bond strength compared to alumina ones $(2,7,8)$.

Despite the enthusiastic debut, their clinical application revealed that alumina brackets are prone to higher incidence of bracket failure during orthodontic treatment and at debonding process, whilst their most important complication soon surfaced in the form of iatrogenic trauma on enamel during debonding $(4,9)$. The aforementioned complications have been appended to the brittle nature of ceramics, which combined with low $\mathrm{K}_{\mathrm{ic}}$, which indicates a low resistance to crack propagation (10), lead to an unfavorable response to loading during orthodontic treatment. In as much, enamel crack formation during debonding, drew the attention of many studies which adopted various approaches ranging from engineering to epidemiology of complications. The percentage of the ceramic bracket base pad covered by enamel during debonding was found to range as much as 3 times higher than the corresponding rate of debonded metallic bracket. The extent of damage for alumina brackets was found up to $46 \%$, which however may be an underestimation as fragments of enamel may not be retained by adhesive on the base bracket pad (11).

Although iatrogenic damage of enamel is the most serious complication, the fracture of alumina brackets during orthodontic therapy $(12,13)$ or debonding is considered also as a major clinical complication prolonging the treatment (14-16). In addition, the removal of broken alumina brackets is difficult and requires extensive chair time for polishing (17) while the use of a rubber dam is recommended (18). This procedure is carried out by using diamond stones to remove the remaining 
part of alumina bracket (19) jeopardizing the enamel integrity (15). Based on clinical data, a study has shown that alumina bracket have a fracture rate of $14.6 \%$ during debonding alone (11). However, a fractographic analysis of fractures of single-crystal alumina brackets occurring during treatment, reported that internal defects was the primary cause of fracture $(47.5 \%)$, followed by external defects induced by machining the single-crystal during manufacturing (42.5\%) (13).

Given that the mechanism of fracture in ceramics is associated with the propagation of internal cracks, it is rational to assume that this phenomenon will be facilitated under intraoral ageing. The Griffiths equation describes the relationship between applied nominal stress and crack length at fracture, i.e. when it becomes energetically favourable for a crack to grow. When a brittle body is subjected to loading and experiences a crack propagation, there is a change of energy taking place which comprises of changes in the potential energy of the body and the surface energy of the new fracture surfaces.

The foregoing theory predicts that the decreased surface energy associated with immersing ceramics in the intraoral environment would cause a reduction in the magnitude of the critical stress that is the stress required to introduce fracture. This degradation in structure integrity should be reflected in mechanical properties of used brackets and thus the aim of this study was to test if the mechanical properties of alumina brackets show inferior characteristics after intraoral exposure. The null hypothesis tested was that no significant differences will be found between the as received and clinically-aged alumina brackets.

\section{MATERIALS AND METHODS}

\section{Sample collection}

Two brands of commercially available alumina brackets were included in this study: a single-crystal (Radiance, American Orthodontics, Sheboygan, WI) and a polycrystalline one (Clarity, 3M, St. Paul, 
MN). Ten brackets from each brand were collected from different patients after exposure in the oral cavity of patients for at least 3 months; another group of ten specimens per material were used in the as-retrieved (dry) condition serving as control. Briefly, patients undergoing orthodontic treatment received ceramic brackets ligated to wires with elastomeric moduli but not bonded to enamel. The reason for avoiding conventional bracket placement involving bonding was the necessity of retrieving the brackets without applying stresses during debonding and without additional fractures. By bonding the brackets to the enamel, there would be a need to debond them and that means that a stress should have been applied along with potential fractures of the wing. Both these parameters would have adverse effects on measuring the mechanical properties of brackets. For the same reason brackets were ligated under the wire in a way to facilitate clearance from occlusal contacts with opposing teeth. The 3-month period was considered an adequate time frame to initiate wetting and ageing as intraoral ageing has been shown to set early after the placement of materials in the oral cavity. Specifically, relevant research has demonstrated that what is considered ageing in the broader dental ceramic field consists of 3 month immersion (20) in laboratory environment in liquid media which bear no comparison with the actual presence of the brackets in the oral cavity. All patients provided consent and the protocol was approved by the Ethics Committee of....University.

At the best of our knowledge there are not previously published data of mechanical properties tested and thus an assessment of sample size was not feasible. Therefore, sample size calculation which requires that previously published data on the properties tested are used to define the sample size required, was not appropriate to conduct. At any rate, retrieval analysis do not follow this rule because the intraoral aging of materials in different oral cavities of patients does not constitute a standardized treatment on specimens; as a result the emphasis is on the actual clinical conditions and their effects and not on standardization of the effect of ageing (21). 
Brackets from all 4 groups (single-crystal and polycrystalline before and after intraoral exposure) were then subjected to:

\section{Raman spectroscopy}

One Raman spectrum was acquired from the wing region of all brackets tested. The brackets were placed on the stage of a microscope (LEICA BME, Leica microsystems Ltd, Heerbrugg, Switzerland) and the region of interest were determined employing optical lens at $40 \mathrm{X}$ nominal magnification. A special device (MicroViewer-785, Raman Microscope Adaptor) was used to attach the Raman Probe to the microscope. Spectra were acquired with an EZ Raman-I, high sensitivity portable Raman analyzer equipped with a Laser (Soliton, Laser Und Messtechnik, Gliching, Germany) operating at $480 \mathrm{~mW}$ output power, $785 \mathrm{~nm}$ emitted wavelength and a nominal resolution of $4.5-6.5 \mathrm{~cm}^{-1}$.

\section{Instrumented Indentation Testing (IIT) and Fracture Toughness}

All brackets were embedded in epoxy resin and subjected to metallographic grinding and polishing. The mechanical properties of four groups (radiance control: RAC, radiance retrieved RAR, clarity control: CLC and clarity retrieved CLR) were determined using instrumented indentation testing (IIT) according to ISO 14577-2002 (22). The mechanical properties tested were Martens hardness (HM), indentation modulus $\left(\mathrm{E}_{\mathrm{T}}\right)$ and the ratio of elastic to total work, commonly known as elastic index $\left(\eta_{I T}\right)$. Additional information for the methodology and mathematical formulas can be found in recently published papers $(23,24)$ In addition a recently introduced analytical technique was employed for the first time in relevant research, to estimate the fracture toughness of orthodontic brackets combining the results of IIT and crack length around Vickers indentation (25). The first step is the acquisition of force indentation depth curve with an instrumented indentation tester equipped with Vickers indenter at a preselected maximal load, $\mathrm{P}_{\mathrm{m}}$. Then the nominal half diagonal length (a) and nominal hardness $\left(H_{n}\right)$ were calculated according to the following equations:

$$
a=3.5 h_{m}(1) \text { and } H_{n}=P_{m} /\left(24.5 h_{m}{ }^{2}\right)
$$


where $h_{m}$ stands for the maximum indentation depth.

The elastic index is determined by dividing the elastic $\mathrm{W}_{\mathrm{e}}$ to total work $\mathrm{W}_{\mathrm{t}}$; both of them are calculated by integrating the areas under the unloading and loading curves, respectively. The length of four cracks originated from the four corners of impression (Fig. 3) were measured and the average length was determined as $\mathrm{c}=\left(\mathrm{c}_{1}+\mathrm{c}_{2}+\mathrm{c}_{3}+\mathrm{c}_{4}\right) / 4$. Then one of the following conditions must be satisfied:

$1.5 \leq \mathrm{c} / \mathrm{a} \leq 6$ with $0.3 \leq \mathrm{W}_{\mathrm{e}} / \mathrm{W}_{\mathrm{t}} \leq 0.71$

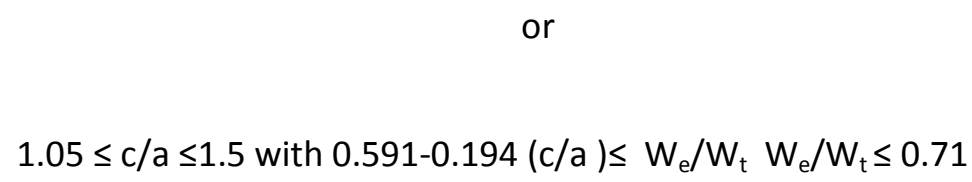

Should neither of the aforementioned conditions was satisfied, a different Pm must be selected and the abovementioned procedure must be repeated. The modulus of elasticity $E$ is given by the formula:

$$
E=\frac{1-v^{2}}{\frac{\sum_{m=1}^{6}\left[a_{m}\left(\frac{W_{e}}{W_{t}}\right)^{m}\right]}{H_{n}}-\frac{1.32\left(1-v_{i}^{2}\right)}{E_{i}}}
$$

where:

$E_{i}(1141 \mathrm{GPa})$ and $\mathrm{v}_{\mathrm{i}}(0.07)$ are the modulus of elasticity and Poisson ratio of Vickers indenter, respectively,

$v(0.22)$ the Poisson ratio of $\mathrm{Al}_{2} \mathrm{O}_{3}$, and

$a_{1}=0.170204, a_{2}=-0.157669, a_{3}=0.110937, a_{4}=-0.048401, a_{5}=-0.005516$ and $a_{6}=0.007625$.

Then $\eta$ is provided by the formula:

$$
\eta=\frac{E /\left(1-0 . v^{2}\right)}{E_{i} /\left(1-v_{i}^{2}\right)}
$$


Finally, the fracture toughness $\mathrm{K}_{\mathrm{IC}}$ is calculated by the formula:

$$
K_{I C}=\left(\frac{10^{-6} P_{m}}{c^{\frac{3}{2}}}\right) * \frac{1+\eta\left[\left(0.0068\left(\frac{c}{a}\right)^{2}-0.1118\left(\frac{c}{a}\right)+0.8295\right]\right.}{0.0757\left(\frac{W_{e}}{W_{t}}\right)^{2}-0.1956\left(\frac{W_{e}}{W_{t}}\right)+0.1285}
$$

where: the unit $\mathrm{K}_{\mathrm{IC}}$ is in MPa $\mathrm{m}^{1 / 2}$, length of $\mathrm{c}$ and $\mathrm{a}$ is in meters, and force is expressed in Newton.

\section{Statistical analysis}

The results of $H M, E_{I T}, \eta_{I T}$ and $K_{I C}$ were statistically analyzed by two way ANOVA employing brackets and condition as discriminating variables while significant differences were identified by Tukey multiple comparison test at $a=0.05$. The normality and homoscedasticity of all data were initially checked by Kolmogorov-Smirnov and equal test variance respectively.

\section{RESULTS}

\section{Raman spectroscopy}

Figure 1 demonstrates representative Raman spectra from all groups tested. The characteristic peaks at $379,418,429,578,644$ and $750 \mathrm{~cm}^{-1}$ correspond to $\alpha-\mathrm{Al}_{2} \mathrm{O}_{3}$, which is the most common form of crystalline aluminum oxide (corundum). Both brackets showed identical spectra before and after in vivo ageing.

\section{Instrumented Indentation Testing (IIT) and Fracture Toughness}

Figure 2 illustrates representative force-indentations depth curves from all groups tested. It may be worth noting that despite the variations found within groups, curves from same bracket demonstrated good reproducibility. All data were drawn from normally distributed populations and share equal standard deviations as determined after Kolmogorov-Smirnov and equal variance tests $(p>0.05)$. 
In Figure 3 representative images of Vickers impression from the surfaces of both brackets are presented. All cracks are originated from the four corners of Vickers impression as expected for polycrystalline alumina bracket (Clarity)(Figure $3 \mathrm{~A})$. In contrast, an irregular crack distribution pattern was found for the single crystal alumina bracket (Radiance) (Figure 3B), which precluded the measurement of $K_{\mathrm{I}}$, because of the inability to define crack lengths.

The mean values and standard deviations of $H M, E_{I T}, \eta_{I T}$ and $K_{I C}$ are presented in Figure 4. No statistically significant differences were found between brackets or between before and after in vivo ageing with the exception of $\eta_{\text {IT }}$ between brackets (Figure 4). The effect of different brackets (singlecrystal vs. polycrystalline) did not seem to be depended on the treatment or state (control vs. in vivo aged) as there was no statistically significant interaction between brackets and condition $(p>0.05)$ for all properties tested.

\section{DISCUSSION}

Based on the results of this study the null hypothesis must be accepted as no significant differences were found between the control and retrieved alumina brackets tested.

The usefulness of Raman analysis as applied to the study of the specimens was twofold: it aided to identify the structure of alumina and allocates possible differences between single-crystal and polycrystalline brackets and also it assists in excluding the possibility that differences in mechanical properties are related to phase transformation such in case of tetragonal stabilized zirconia (26). However both brackets were found to consists of the stable corundum alumina phase and the structure of both brackets is unaffected by intraoral aging as shown in Fig 1.

All mechanical properties studied ( $\mathrm{HM}, \mathrm{E}_{\mathrm{IT}}, \eta_{\mathrm{IT}}$ and $\left.\mathrm{K}_{\mathrm{IC}}\right)$ showed no significant differences before and after intraoral aging implying that, contrary to what is predicted by the theoretical model, the mechanical properties are not affected by the short-term intraoral exposure. In addition, the 
findings indicated that both bracket groups have similar mechanical properties (apart from elastic index), a finding which is appended to the fact that they are made of the same material. The higher elastic index of single crystal bracket (Clarity) denotes a more brittle material compared to polycrystalline one (Radiance). To the best of authors' knowledge there has been no previously reported assessment of the properties reported in this study and thus no comparison can be made with data derived from published studies as those were just provided data of the raw material (alumina) derived from relevant tables found in technical bulletins.

From the early introduction of ceramic brackets to the profession, the paramount importance of $\mathrm{K}_{\mathrm{IC}}$ in clinical behavior was highlighted $(6,10,27)$. Given that $K_{1 C}$ of alumina is considered 20 to 40 times lower than stainless steel $(13,28)$, the fracture of alumina brackets is more likely to occur compared to metallic ones. Although a range of values have been given for single-crystal and polycrystalline alumina brackets, these values have never been measured experimentally as noted previously. The reason was that $K_{I C}$ measurement requires bulky specimens with special notches (25) which are impossible to be induced in brackets because of the size and complex morphology of orthodontic appliances. An alternative is to measure the $\mathrm{K}_{\mathrm{IC}}$ with the indentation method, which has been widely used for this purpose mainly due to superiority in efficiency and convenience (25).

$\mathrm{K}_{\mathrm{IC}}$ for the polycrystalline Clarity was found to be $1.9 \mathrm{MPa} \mathrm{m}{ }^{1 / 2}$, which is lower than the value of 99.9\% hot-pressed or sintered, pure, fine-grained alumina (3-4 MPa $\left.\mathrm{m}^{1 / 2}\right)$, sintered or glass-bonded alumina (2.5-5 MPa $\left.\mathrm{m}^{1 / 2}\right)$, or coarse-grained alumina (3-5 MPa $\left.\mathrm{m}^{1 / 2}\right)(29)$. This lower value may be attributed to material quality and/or manufacturing processes, which is considered proprietary. Testing of the single-crystal alumina bracket (Radiance) provided an irregular pattern of cracks around Vickers indentations, which is typical for single-crystal alumina as the crack propagation is dominated by crystal orientation (30). The estimation of $\mathrm{K}_{\mathrm{IC}}$ in this case was not feasible and this is might constitute a limitation of the indentation technique, reported for the first time in the application of this methodology to orthodontic ceramics. 
The calculation of $K_{1 c}$ gives the ability to estimate the critical size of crack $(\alpha)$ which would lead to catastrophic failure according to equation (10):

$$
a=\frac{K_{I C}^{2}}{\pi * \sigma_{F}^{2}}=0.6 \mu m(7)
$$

where $\sigma_{\mathrm{F}}$ is the fracture strength.

The abovementioned value is almost 10 times lower than the $5.5 \mu \mathrm{m}$ critical crack size (10), which has been calculated employing the most widely reported value of $\mathrm{K}_{\mathrm{IC}}\left(5.8 \mathrm{MPa} \mathrm{\textrm {m } ^ { 1 / 2 }}\right)$. This implies that the material may be more crack susceptible than previously considered, since even minute cracks can lead to catastrophic failure if loaded at $\sigma_{\mathrm{F}}$. The aforementioned calculations also demonstrate the reason for the tremendous impact of this property on performance of brittle materials, and explain the frequent fracture of ceramic brackets during routine orthodontic mechanics, especially at debonding.

Fracture mechanics was developed during World War I by an English aeronautical engineer Griffith, hence the term Griffith crack, to explain the failure of brittle materials, which showed a paradox: the stress required to fracture bulk glass ranged around $100 \mathrm{MPa}$, whereas the theoretical stress estimated to fracture it was 100 times higher. Therefore, there was a necessity to introduce a theory to explain the contradicting evidence. Griffith suggested that the low fracture strength observed in experiments, as well as the size-dependence of strength, was due to the presence of microscopic flaws in the bulk material. Because the expression derived by Griffith's work provided the critical stress as a function of the surface energy through critical surface tension of the material, and since this term is reduced when the brittle material is wetted, the critical stress required to induce failure was hypothesized to be reduced at wet conditions. Although this has been already demonstrated in dental ceramics $(31,32)$, it was not verified in the case of ceramic brackets mainly because the effect of internal defects in the form of cracks arising from the manufacturing of ceramic brackets, seem to modify the loading pattern and initiation of stress-induced cracks. 
The results of this study have also significant clinical implications. Given that none of the tested properties showed a reduction after intraoral exposure, the fracture of orthodontic brackets during deboning is not associated with the ageing-related inferior mechanical properties but probably with variations in their mechanical properties as a result of manufacturing-induced crack formation and distribution in the material.

\section{Conclusions}

The mechanical properties of single crystal and polycrystalline alumina brackets are not affected by exposure to a wet environment in contrast to the prediction of theoretical mode, possibly because of the formation of internal cracks during the manufacturing process.

Single crystal and polycrystalline brackets share equal mechanical Martens hardness and Elastic Modulus but single crystal demonstrated higher brittleness.

Funding: no funding was obtained for this study from any source. 


\section{REFERENCES}

1.Harris, A. M. P., Joseph, V. P., Rossouw, P. E. (1992) Shear peel bond strengths of esthetic orthodontic brackets. American Journal of Orthodontics and Dentofacial Orthopedics, 102, 215-219.

2.Eliades, T., Eliades, G., Brantley, W. (2001) Orthodontic Brackets. In: Brantley W. ET (ed.). Orthodontic Materials, Thieme, Stuttgard, pp. 151-169.

3.Eliades, T. (2007) Orthodontic materials research and applications: Part 2. Current status and projected future developments in materials and biocompatibility. American Journal of Orthodontics and Dentofacial Orthopedics, 131, 253-262.

4.Winchester, L. J. (1991) Bond Strengths of 5 Different Ceramic Brackets - an Invitro Study. European Journal of Orthodontics, 13, 293-305.

5.Karamouzos, A., Athanasiou, A. E., Papadopoulos, M. A. (1997) Clinical characteristics and properties of ceramic brackets: A comprehensive review. American Journal of Orthodontics and Dentofacial Orthopedics, 112, 34-40.

6.Bishara, S. E., Fehr, D. E. (1997) Ceramic brackets: something old, something new, a review. Semin Orthod, 3, 178-188.

7.Keith, O., Kusy, R. P., Whitley, J. Q. (1994) Zirconia Brackets - an Evaluation of Morphology and Coefficients of Friction. American Journal of Orthodontics and Dentofacial Orthopedics, 106, 605614.

8.Springate, S. D., Winchester, L. J. (1991) An evaluation of zirconium oxide brackets: a preliminary laboratory and clinical report. British Journal of Orthodontics, 18, 203-209.

9.Jeiroudi, M. T. (1991) Enamel Fracture Caused by Ceramic Brackets. American Journal of Orthodontics and Dentofacial Orthopedics, 99, 97-99.

10.Kusy, R. P. (1988) Morphology of Polycrystalline Alumina Brackets and Its Relationship to Fracture-Toughness and Strength. Angle Orthodontist, 58, 197-203.

11.Cochrane, N. J., Lo, T. W. G., Adams, G. G., Schneider, P. M. (2017) Quantitative analysis of enamel on debonded orthodontic brackets. American Journal of Orthodontics and Dentofacial Orthopedics, 152, 312-319.

12.Gibbs, S. L. (1992) Clinical performance of ceramic brackets: a survey of British orthodontists' experience. British Journal of Orthodontics, 19, 191-197.

13.Viazis, A. D., Chabot, K. A., Kucheria, C. S. (1993) Scanning Electron-Microscope (Sem) Evaluation of Clinical Failures of Single-Crystal Ceramic Brackets. American Journal of Orthodontics and Dentofacial Orthopedics, 103, 537-544.

14.Rhodes, R. K., Duncanson, M. G., Nanda, R. S., Currier, G. F. (1992) Fracture Strengths of Ceramic Brackets Subjected to Mesial-Distal Archwire Tipping Forces. Angle Orthodontist, 62, 67-75.

15.Matasa, C. G. (1999) Impact resistance of ceramic brackets according to ophthalmic lenses standards. American Journal of Orthodontics and Dentofacial Orthopedics, 115, 158-165.

16. Holt, M. H., Nanda, R. S., Duncanson, M. G. (1991) Fracture-Resistance of Ceramic Brackets during Arch Wire Torsion. American Journal of Orthodontics and Dentofacial Orthopedics, 99, 287-293. 17.Wool, A. L. (1992) A Better Debonding Procedure. American Journal of Orthodontics and Dentofacial Orthopedics, 102, 84-86.

18.Chate, R. A. C. (1993) Safer Orthodontic Debonding with Rubber Dam. American Journal of Orthodontics and Dentofacial Orthopedics, 103, 171-174.

19.Britton, J. C., Mcinnes, P., Weinberg, R., Ledoux, W. R., Retief, D. H. (1990) Shear Bond Strength of Ceramic Orthodontic Brackets to Enamel. American Journal of Orthodontics and Dentofacial Orthopedics, 98, 348-353.

20. Drummond, J.L., King, T.J., Bapna, M.S., Koperski, R.D. (2000) Mechanical property evaluation of pressable restorative ceramics. Dental Materials 16, 226-33.

21. Eliades T, Eliades G, Brantley WA. Watts DC (2005) In vivo ageing of dental materials: aging and related phenomena. Quintessence, Chicago 2005.

22.ISO14577-1 2002 Metallic materials - Instrumented indentation test for hardness and materials parameters. International Organization for Standardization, Geneva 
23.Zinelis, S., Al Jabbari, Y. S., Gaintantzopoulou, M., Eliades, G., Eliades, T. (2015) Mechanical properties of orthodontic wires derived by instrumented indentation testing (IIT) according to ISO 14577. Prog Orthod, 16, 19.

24.Zinelis, S., Sifakakis, I., Katsaros, C., Eliades, T. (2014) Microstructural and mechanical characterization of contemporary lingual orthodontic brackets. European Journal of Orthodontics, 36, 389-393.

25.Ma, D., Wang, J., Sun, L. (2017) Methodology for measuring fracture toughness of ceramic materials by instrumented indentation test with vickers indenter. Journal of the American Ceramic Society, 100, 2296-2308.

26.Zhuang, Y. X., Zhu, Z. Y., Jiao, T., Sun, J. (2019) Effect of Aging Time and Thickness on LowTemperature Degradation of Dental Zirconia. Journal of Prosthodontics-Implant Esthetic and Reconstructive Dentistry, 28, E404-E410.

27.Scott, G. E. (1988) Fracture-Toughness and Surface Cracks - the Key to Understanding Ceramic Brackets. Angle Orthodontist, 58, 5-8.

28.Ghafari, J. (1992) Problems associated with ceramic brackets suggest limiting use to selected teeth. Angle Orthodontist, 62, 145-152.

29.Quin, G. (2007) Fractography of ceramic and glasses National Institute of Standards and Technology, Washington

30.Wang, K., Jiang, F., Yan, L., Xu, X., Wang, N., Zha, X., Lu, X., Wen, Q. (2019) Study on mechanism of crack propagation of sapphire single crystals of four different orientations under impact load and static load. Ceramics International, DOI: 10.1016/j.ceramint.2019.01.021

31.Drummond, J. L., Novickas, D., Lenke, J. W. (1991) Physiological aging of an all-ceramic restorative material. Dental Materials, 7, 133-137. 
Figures

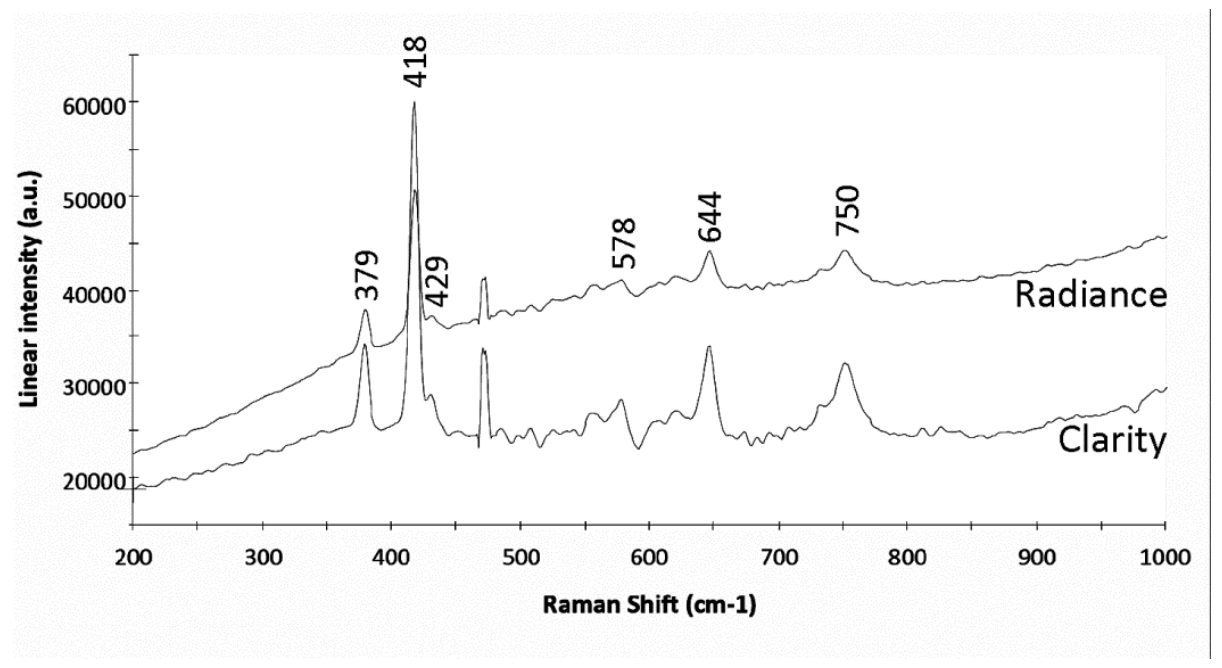

Figure 1. Representative Raman spectra of as received and retrieved ceramic brackets. For the sake of clarity only one spectrum is presented for each bracket.

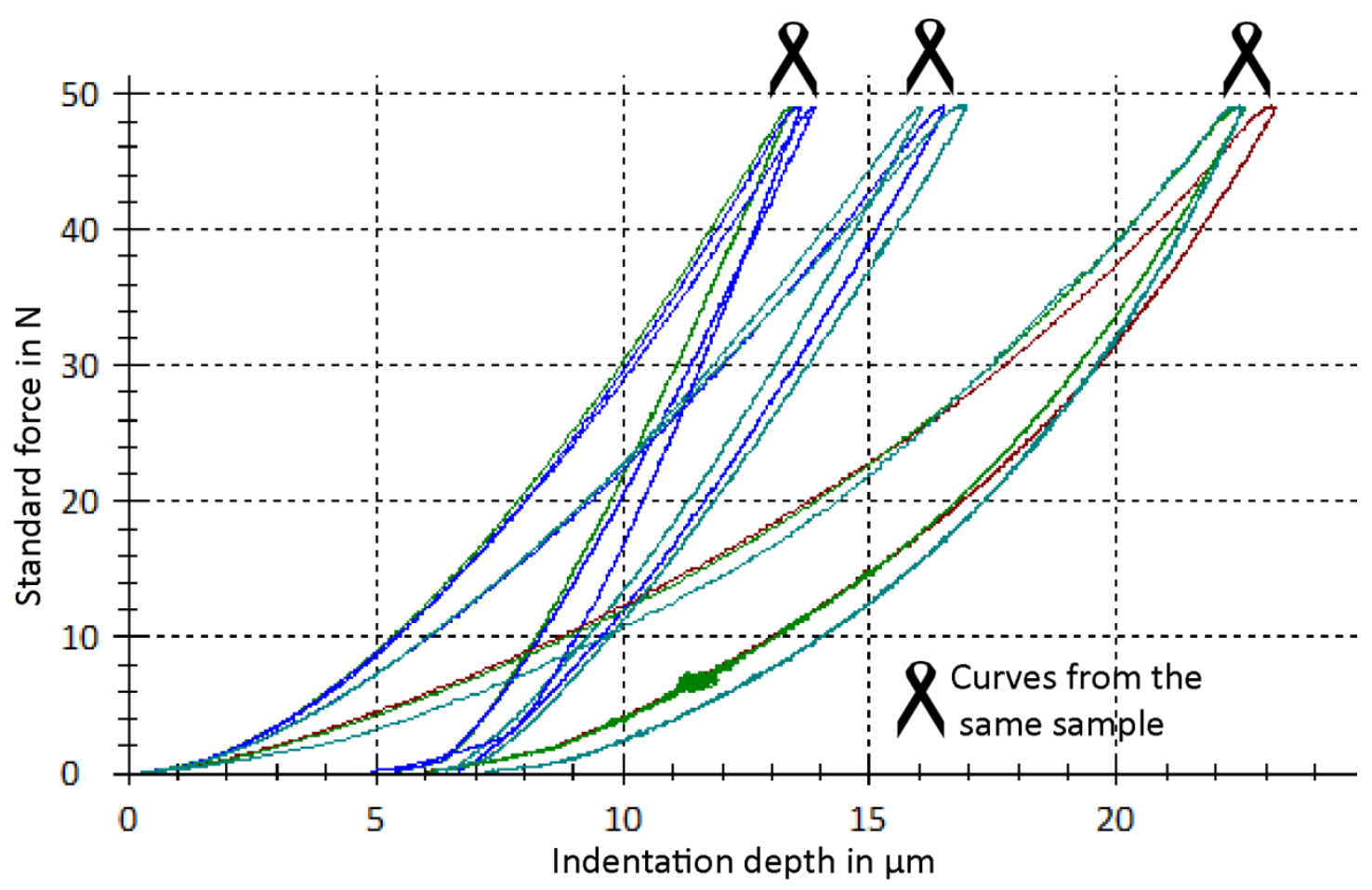


Figure 2. Representative force indentation depth curves from all groups tested. The curves from each bracket showed good reproducibility but all the groups showed great variation in tested mechanical properties.

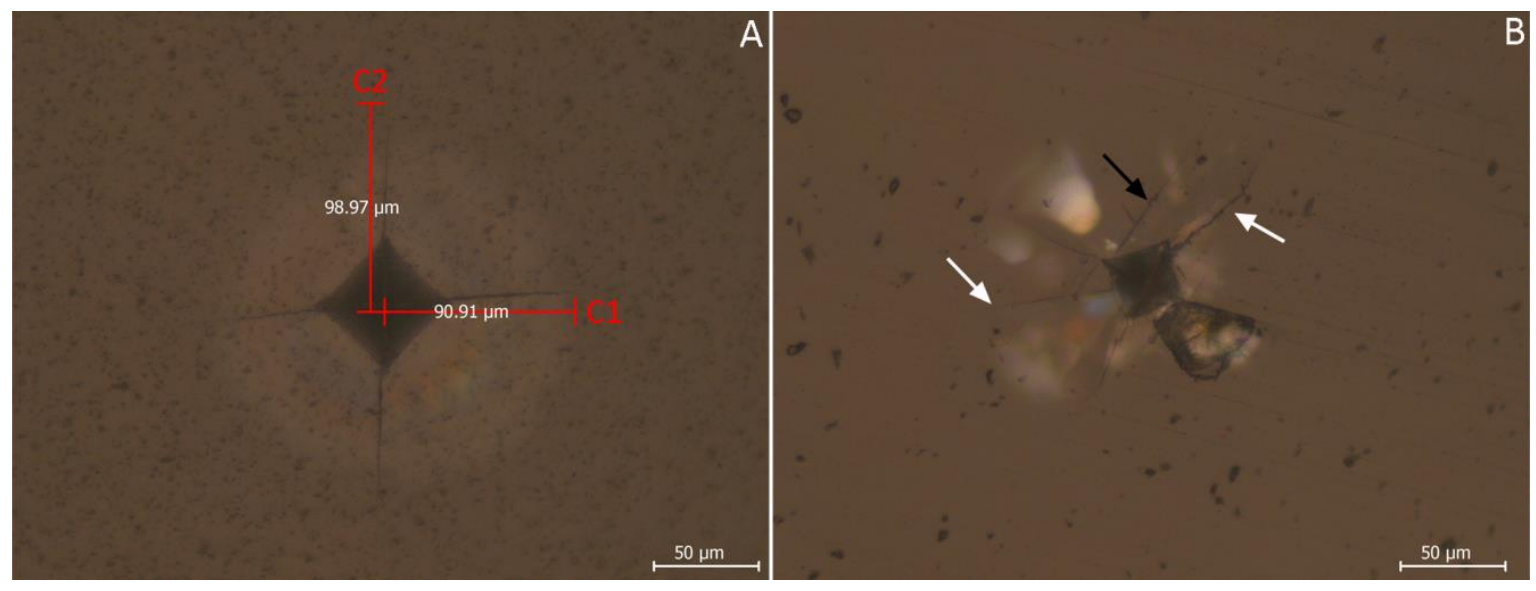

Figure 3. A) Representative image of Vickers impression from the surface of Clarity. All cracks are originated from the four corners of Vickers impression. The $\mathrm{C} 1$ and $\mathrm{C} 2$ denote the crack length from the center of impression. The other two ( $\mathrm{C} 3$ and $\mathrm{C} 4$ ) are not shown for the sake of clarity. B) Representative image from the surface of Radiance. The longest cracks are not originated from the corners of impression (white arrows) while a crack perpendicular to the left corner is presented. Chipping of adjacent area at the right corner is easily identified (bar: $50 \mu \mathrm{m}$ ). 

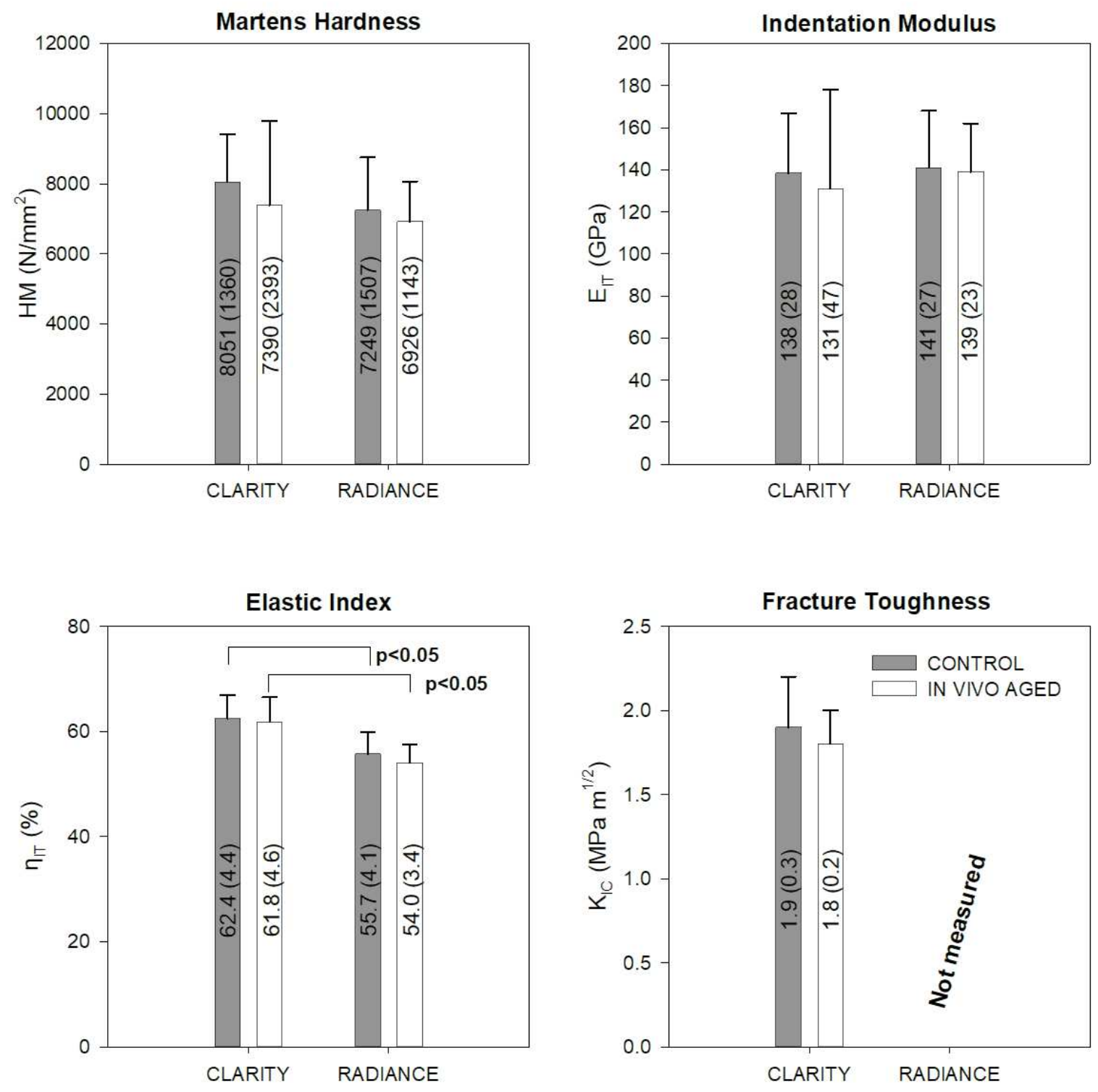

Figure 4. Mean values and standard deviations of all properties tested. Numerical values are presented within the bars. Horizontal lines connect mean values with significant statistical differences. 